Article

\title{
Effect of Surface Modifications of SBA-15 with Aminosilanes and 12-Tungstophosphoric Acid on Catalytic Properties in Environmentally Friendly Esterification of Glycerol with Oleic Acid to Produce Monoolein
}

\author{
Kullatida Ratchadapiban ${ }^{1}$, Piyasan Praserthdam ${ }^{2}$, Duangamol Nuntasri Tungasmita ${ }^{3}$, \\ Chutima Tangku ${ }^{1}$ and Wipark Anutrasakda ${ }^{4, *}$ \\ 1 Department of Chemistry, Faculty of Science, Chulalongkorn University, Payathai Road, Patumwan, \\ Bangkok 10330, Thailand; r.kullatida@hotmail.com (K.R.); tang.chutima38@gmail.com (C.T.) \\ 2 Center of Excellence on Catalysis and Catalytic Reaction Engineering, Department of Chemical Engineering, \\ Faculty of Engineering, Chulalongkorn University, Payathai Road, Patumwan, Bangkok 10330, Thailand; \\ piyasan.p@chula.ac.th \\ 3 Center of Excellence in Catalysis for Bioenergy and Renewable Chemicals (CBRC), Department of Chemistry, \\ Faculty of Science, Chulalongkorn University, Payathai Road, Patumwan, Bangkok 10330, Thailand; \\ duangamol.n@gmail.com \\ 4 Green Chemistry for Fine Chemical Productions STAR, Department of Chemistry, Faculty of Science, \\ Chulalongkorn University, Payathai Road, Patumwan, Bangkok 10330, Thailand \\ * Correspondence: wipark.a@chula.ac.th; Tel.: +66-2-218-7622
}

Received: 1 August 2018; Accepted: 24 August 2018; Published: 28 August 2018

\begin{abstract}
A series of protonated amino-functionalized SBA-15 materials was synthesized and tested as heterogeneous catalysts for the esterification of glycerol with oleic acid to produce monoolein. Mesoporous SBA-15 (S) was functionalized with three different aminosilanes: 3-aminopropyltriethoxysilane $\left(\mathrm{N}^{1}\right)$; [3-(2-amino-ethylamino)propyltrimethoxysilane] $\left(\mathrm{N}^{2}\right)$; and (3-trimethoxysilylpropyl) diethylenetriamine $\left(\mathrm{N}^{3}\right)$, before being impregnated with $40 \mathrm{wt} \%$ 12-tungstophosphoric acid (HPW). The resulting nanocatalysts (S-N $\mathrm{N}^{\mathrm{x}}-\mathrm{HPW}$ ) were characterized by $\mathrm{X}$-ray powder diffraction $(\mathrm{XRD})$, Fourier transform infrared spectroscopy (FT-IR), $\mathrm{N}_{2}$ adsorption-desorption, SEM equipped with energy dispersive $\mathrm{X}$-ray spectroscopy (SEM-EDX), transmission electron microscopy (TEM), and elemental analysis techniques. The presence of components of the nanocatalysts and the preservation of the hexagonal structure of SBA-15 were confirmed. Using different functionalizing agents considerably affected the textural properties and acidity of the synthesized nanocatalysts, which helped to improve the catalytic performance. In particular, S- $\mathrm{N}^{2}-\mathrm{HPW}$ was more active and selective than other catalysts in this study, as well as than a number of other commercial acid catalysts, with $95.0 \%$ oleic acid conversion and $60.9 \%$ monoolein selectivity being obtained after five $\mathrm{h}$ of reaction at $160{ }^{\circ} \mathrm{C}$ using $2.5 \mathrm{wt} \%$ of catalyst loading and glycerol/oleic acid molar ratio of 4:1. Aminosilane functionalization also helped to increase the reusability of the catalysts to at least six cycles without considerable loss of activity through strong electrostatic interactions between HPW anions and the protonated amino-functionalized SBA-15 materials.
\end{abstract}

Keywords: amino-functionalized SBA-15; 12-tungstophosphoric acid; acid catalyst; esterification; glycerol 


\section{Introduction}

Glycerol is a by-product of several industrial processes, such as productions of oleochemicals, soap, and biodiesel. Nowadays, the vast majority of glycerol is generated from biodiesel production and, due to the continued rapid growth of the biodiesel industry, the oversupply of glycerol has been a challenging problem for researchers [1]. Efforts have been made to alleviate the demand-supply imbalance, such as the conversion of crude glycerol into high value chemical products [1]. Ester products, in particular, have gained much interest due to their high added value as well as the simplicity and eco-friendliness of the glycerol conversion process [2,3]. To form esters from glycerol, esterification of glycerol with short- and medium-chain organic acids $(C \leq 12)$ has been more commonly performed than with the long-chain counterparts, largely due to the fact that the long alkyl chains may cause steric hindrance and in turn decrease the reactivity with glycerol [2]. Nonetheless, esterification of glycerol with long-chain fatty acids should also be given special attention since, as compared to shorter-chain fatty acids, higher-value products such as monoglycerides can be produced. Essentially, monoglycerides are some of the most important emulsifiers widely used in the food, pharmaceutical, and cosmetic industries [4].

For the conversion of crude glycerol into high-value monoglycerides, two catalyzed processes are commonly used: esterification of glycerol with fatty acids catalyzed by homogeneous strong acids such as sulfuric acid and phosphoric acid; and transesterification of triglycerides with glycerol catalyzed by homogeneous strong bases such as sodium hydroxide and calcium hydroxide [3]. However, since the aforementioned processes involve the use of homogeneous catalysts, undesired characteristics such as low separability and recoverability are to be expected. Using homogeneous catalysts for this purpose also requires high reaction temperature, which in turn elevates the risk of deterioration of taste, aroma, and color of monoglycerides [5]. The development of a more efficient catalytic system for the conversion of glycerol to produce monoglycerides is therefore required, and has so far been a scientific challenge.

In search of a better catalytic system for this purpose, many studies have focused on the use of heterogeneous catalysts mainly because of their superior separability and recoverability. These advantages eliminate the need for a neutralization step and reduce the formation of waste. Recently, some of the most notable heterogeneous catalytic systems for this type of esterification involve the use of ion exchange resins [6], functionalized clays [7], sulfated metal oxides [8], metal-organic frameworks [9], zeolites [10], hydrotalcites [11], and supported heteropolyacids (HPAs) [12].

The use of supported HPAs as catalysts for this type of reaction can be especially advantageous. In particular, as compared to several other types of catalysts such as those based on mineral acids, the Brønsted acidity of HPAs is remarkably higher. So far, more than a hundred varieties of structure and composition of HPAs have been found [13]. Nevertheless, there are only a few types of HPAs whose physicochemical and catalytic properties have been characterized [14]. Keggin-structure HPAs are among those few, which have received special attention due to their simple synthesis and thermal stability. These characteristics can be beneficial for catalyzing the conversion of glycerol to monoglycerides. In particular, 12-tungstophosphoric acid $\left(\mathrm{H}_{3} \mathrm{PW}_{12} \mathrm{O}_{40}, \mathrm{HPW}\right)$ is one of the highly suitable Keggin-structure HPAs for catalyzing this conversion reaction, owing to its high acid strength, low corrosivity, high thermal stability, and environmental friendliness [15].

Using HPW as a heterogeneous catalyst requires the use of a support material since, like other HPAs, HPW is highly soluble in polar solvents. In addition, its surface area is relatively low $\left(<10 \mathrm{~m}^{2} \mathrm{~g}^{-1}\right)$. Several kinds of support materials have been employed for this purpose, including activated carbon [16], zeolite [17], titania [18], and mesoporous silica [19]. Among these support materials, mesoporous silicas such as SBA-15 are especially suitable for being used as a support for HPW due to their high surface area, proper pore size, and high thermal stability [20]. 
By immobilizing HPW on a solid support including SBA-15, catalytic activity can be improved [19]. However, HPW leaching can occur, especially in polar media, due to the fact that the interaction between HPW and its support is generally weak [21]. To overcome this problem, functional groups may be grafted on the support prior to HPW immobilization in order to allow a strong interaction, such as ionic bond, with HPW. Functionalization of aminosilane on SBA-15 can provide this property, which will in turn help to improve the stability and reusability of the HPW-based catalyst system [22,23].

In the present study, we report the synthesis of a series of protonated amino-functionalized SBA-15 materials by functionalizing SBA-15 (S) with three types of aminosilanes that vary in the number of amino groups: 3-aminopropyltriethoxysilane $\left(\mathrm{N}^{1}\right)$; [3-(2-amino-ethylamino)propyl-trimethoxysilane] $\left(\mathrm{N}^{2}\right)$; and (3-trimethoxysilylpropyl) diethylenetriamine $\left(\mathrm{N}^{3}\right)$, before immobilization with HPW. The non-functionalized HPW-immobilized SBA-15 material was also prepared for comparison purpose. The physicochemical properties of the materials were fully characterized. The synthesized materials were tested as catalysts for the solvent-free esterification of glycerol with oleic acid to produce monoolein. Effects of reaction parameters, including reaction temperature, catalyst loading, and molar ratio of glycerol to oleic acid, on the catalytic performance were investigated. Catalyst reusability and catalytic performance comparison with commercial catalysts were also studied. To the best of our knowledge, this is the first study to investigate the effects of amino functionalization, as well as the effects of different numbers of functionalizing amino groups, on the physicochemical and catalytic properties of HPW-immobilized SBA-15 materials in the esterification of glycerol with oleic acid.

\section{Results and Discussion}

\subsection{Materials Characterization}

\subsubsection{X-ray Powder Diffraction (XRD)}

The X-ray diffraction patterns of the as-synthesized materials are shown in Figure 1. At low angles $\left(0.7^{\circ}\right.$ to $\left.5^{\circ}\right)$ (Figure $\left.1 \mathrm{a}\right)$, the XRD pattern of pure SBA-15 showed three peaks at $2 \theta=1.00,1.60$, and 1.84, corresponding to (100), (110), and (200) reflection planes, respectively. The low-angle XRD data of all protonated amino-functionalized SBA-15 (S-N $\left.{ }^{\mathrm{x}}-\mathrm{HPW}\right)$ materials, showing nearly the same pattern as that of SBA-15, are consistent with a well-ordered 2D hexagonal $(P 6 \mathrm{~mm})$ pore arrangement and characteristics of SBA-15 type materials. This finding indicates that the hexagonal structure was preserved after amino-functionalization and HPW immobilization [24]. Nevertheless, as compared to the peaks of pure SBA-15, the XRD patterns of the S-N $\mathrm{N}^{\mathrm{x}}-\mathrm{HPW}$ materials showed a less intense (100) peak and the disappearance of the (110) and (200) peaks. This latter finding is likely driven by the decrease of the long-range ordering of the mesostructure following the filling of amino groups and HPW in the support mesopores [25]. Additionally, the fact that the (100) diffraction peak of the S-N $\mathrm{N}^{\mathrm{x}}-\mathrm{HPW}$ materials slightly shifted to lower angles, which indicates a slight increase in the $d_{100}$ spacing as compared to that of the parent SBA-15, is likely attributable to the slight expansion of the hexagonal unit cell of the SBA-15 framework during the amino-functionalization and HPW immobilization processes [26].

At high angles $\left(15^{\circ}\right.$ to $\left.70^{\circ}\right)$ (Figure $1 \mathrm{~b}$ ), the XRD patterns of SBA-15 and S-N ${ }^{\mathrm{x}}$-HPW showed only a broad peak at $2 \theta=23^{\circ}-26^{\circ}$, which is characteristic of amorphous silica, and the characteristic XRD peaks of crystalline HPW were not observed. This finding indicates a good dispersion of HPW inside the support mesopores [27]. For the non-functionalized S-HPW, the low-angle and high-angle XRD patterns were similar to those of $\mathrm{S}-\mathrm{N}^{\mathrm{x}}-\mathrm{HPW}$, thus similarly indicating the preservation of the hexagonal structure and the good dispersion of HPW nanoparticles. 

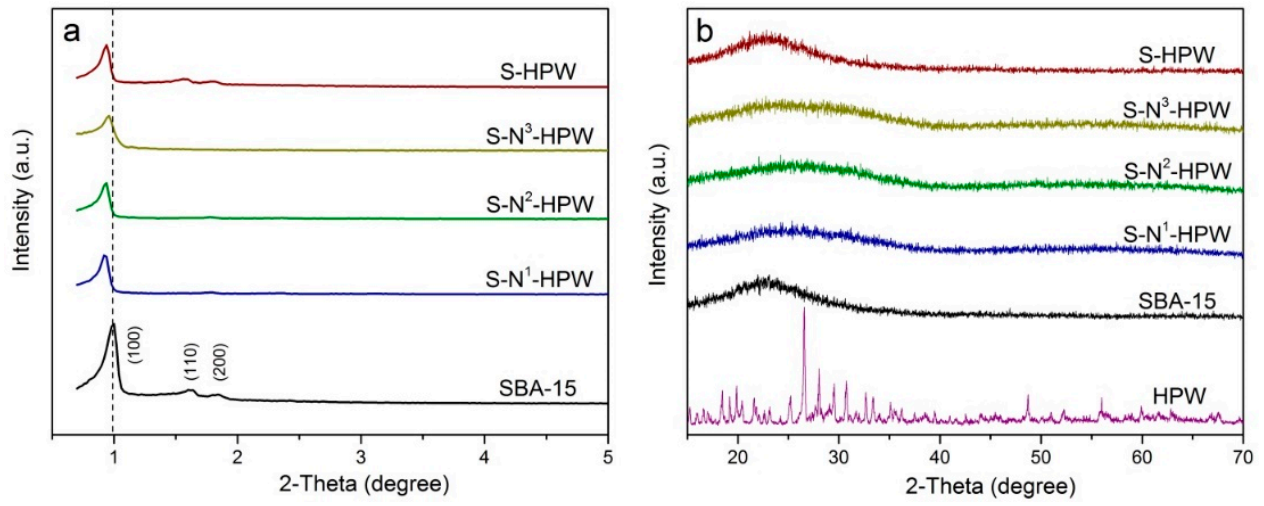

Figure 1. X-ray powder diffraction (XRD) patterns of SBA-15 (S), 12-tungstophosphoric acid (HPW), S-N ${ }^{\mathrm{x}}-\mathrm{HPW}$, and S-HPW materials at low angles (a) and high angles (b).

\subsection{2. $\mathrm{N}_{2}$ Adsorption-Desorption}

$\mathrm{N}_{2}$ adsorption-desorption analysis was used to investigate the surface area and pore characteristics of the synthesized materials, where the pore structure parameters were obtained by the Brunauer-Emmett-Teller (BET), Barrett-Joyner-Halenda (BJH), and t-plot methods. Figure 2a shows $\mathrm{N}_{2}$ sorption isotherms of SBA-15 and its modified analogs. All of the isotherms were classified as type IV isotherms with $\mathrm{H} 1$ hysteresis loops, a typical characteristic of mesoporous materials according to the International Union of Pure and Applied Chemistry (IUPAC) classification [28]. This finding indicates that the structure of the mesoporous silica support was preserved after aminosilane functionalization and HPW immobilization. Furthermore, it was found that the relative pressure of capillary condensation and the hysteresis loop of pure SBA- 15 were higher and wider, respectively, than those of S- $\mathrm{N}^{\mathrm{x}}-\mathrm{HPW}$ samples. This finding suggests the presence of partial blockage of mesopores induced by aminosilane functionalization and HPW immobilization, which is similar to the findings of several previous reports $[22,23,29]$.
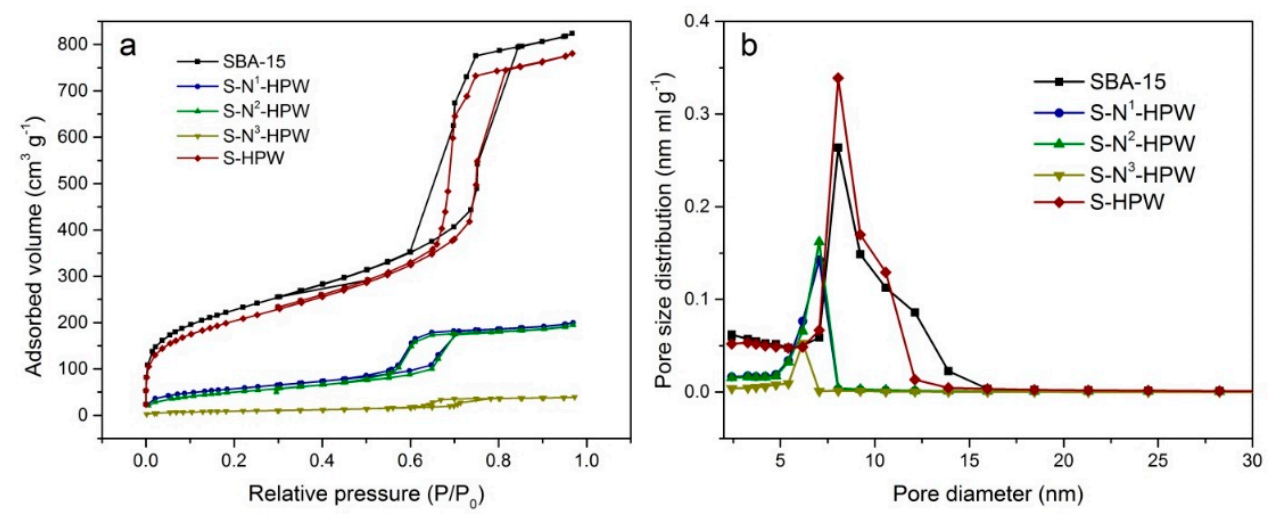

Figure 2. $\mathrm{N}_{2}$ adsorption-desorption isotherms (a) and the corresponding pore size distributions (b) of SBA-15, S-Nx-HPW, and S-HPW materials.

The surface area and pore parameters are reported in Table 1. The pure SBA-15 material was found to have a total surface area of $815 \mathrm{~m}^{2} \mathrm{~g}^{-1}$, an average pore diameter of $8.06 \mathrm{~nm}$, and a pore volume of $1.18 \mathrm{~cm}^{3} \mathrm{~g}^{-1}$. The surface area of the acid-immobilized amino-functionalized SBA-15 materials decreased with respect to that of the pure SBA-15. In particular, functionalization with mono-aminosilane (APTES) and di-aminosilane (AAPTMS) to produce S-N ${ }^{1}-\mathrm{HPW}$ and S-N $\mathrm{N}^{2} \mathrm{HPW}$ led to a total surface area of 205 and $177 \mathrm{~m}^{2} \mathrm{~g}^{-1}$, respectively. For functionalization with tri-aminosilane (DETTMS) to produce S-N ${ }^{3}-\mathrm{HPW}$, the decrease was especially marked where the total surface area was 
found to be $55 \mathrm{~m}^{2} \mathrm{~g}^{-1}$. The latter case might result from a higher degree of deposition of protonated amino groups and HPW anions driven by the greater abundance of amino groups.

While the decrease in total surface area of the modified SBA-15 materials may be attributed to the deposition of protonated amino groups and HPW anions on both the external and internal surfaces of SBA-15 [30], further investigation indicated that such deposition occurred mostly inside the mesopores of SBA-15. Specifically, the internal surface area of the acid-immobilized amino-functionalized SBA-15 samples exhibited a more pronounced decrease as compared to corresponding decrease in external surface area. The successful aminosilane grafting and HPW deposition inside the mesoporous channels of SBA-15 is also evidenced by the fact that aminosilane functionalization and HPW immobilization led to a decrease in total pore volume and pore size of the materials.

Table 1. Physical and chemical properties of SBA-15, S-N $\mathrm{N}^{\mathrm{x}}-\mathrm{HPW}$, and S-HPW materials.

\begin{tabular}{|c|c|c|c|c|c|c|c|c|}
\hline Materials & $\begin{array}{l}\text { Total BET } \\
\text { Surface } \\
\text { Area } \\
\left(\mathrm{m}^{2} \mathrm{~g}^{-1}\right)\end{array}$ & $\begin{array}{c}\text { Internal } \\
\text { Surface } \\
\text { Area }^{\mathrm{b}} \\
\left(\mathrm{m}^{2} \mathrm{~g}^{-1}\right)\end{array}$ & $\begin{array}{c}\text { External } \\
\text { Surface } \\
\text { Area }^{b} \\
\left(\mathrm{~m}^{2} \mathrm{~g}^{-1}\right)\end{array}$ & $\begin{array}{c}\text { Pore } \\
\text { Diameter } \\
\quad(n m)\end{array}$ & $\begin{array}{c}\text { Pore } \\
\text { Volume } \\
\left(\mathrm{cm}^{3} \mathrm{~g}^{-1}\right)\end{array}$ & $\begin{array}{c}\text { Nitrogen } \\
\left(\text { mmole }^{-1}\right)\end{array}$ & $\begin{array}{c}\text { Acidity } \\
\left(\mathrm{mmole}^{\mathrm{e}}\right)^{-1}\end{array}$ & $\begin{array}{l}\text { Tungsten } \mathrm{f} \\
\text { (wt \%) }\end{array}$ \\
\hline S-N ${ }^{1}-\mathrm{HPW}$ & 205 & 151 & 19 & 7.05 & 0.33 & 1.34 & 0.32 & 23.8 \\
\hline S-N ${ }^{2}-\mathrm{HPW}$ & 177 & 116 & 20 & 7.05 & 0.30 & 1.78 & 0.41 & 27.9 \\
\hline S-N ${ }^{3}-\mathrm{HPW}$ & 55 & 28 & 10 & 6.18 & 0.06 & 2.87 & 0.47 & 30.1 \\
\hline
\end{tabular}

${ }^{a}$ From BET desorption method. ${ }^{b}$ From $t$-plot method. ${ }^{c}$ From BJH desorption method. ${ }^{d}$ From elemental analysis.

${ }^{\mathrm{e}}$ From titration with $\mathrm{NaOH}(\mathrm{n}=4) .{ }^{\mathrm{f}}$ From ICP-OES.

The pore size distributions of the synthesized materials calculated from the $\mathrm{BJH}$ desorption method are shown in Figure 2b. All distributions are narrow and indicate that the pore diameters are in the range of $4-6 \mathrm{~nm}$. The mean pore diameters of SBA-15, S-N ${ }^{1}-\mathrm{HPW}, \mathrm{S}-\mathrm{N}^{2}-\mathrm{HPW}$, and S-N ${ }^{3}-\mathrm{HPW}$ samples were $8.06,7.05,7.05$, and $6.18 \mathrm{~nm}$, respectively. This result is in line with the above finding that aminosilane functionalization and HPW immobilization led to partial blockage of mesopores. This finding is also consistent with the previous finding that such a blockage could be caused by grafted aminosilanes, especially the long-chain tri-aminosilane (DETTMS), and relatively bulky HPW anions [19]. However, the fact that pore size distributions and mean pore diameters of S-N ${ }^{1}-H P W$ and S-N ${ }^{2}-\mathrm{HPW}$ were similar to each other but different from those of S-N ${ }^{3}-\mathrm{HPW}$ suggests that using aminosilanes with different amounts of amino groups affected the pore size distribution differently compared to the effects on surface area, essentially since the surface area was significantly different for each acid-immobilized amino-functionalized material. At any rate, changes in pore characteristics following the modifications of SBA-15 would later prove to be an important factor affecting the selectivity of glyceride products when the modified materials are used as catalysts for the glycerol esterification.

\subsubsection{Fourier Transform Infrared Spectroscopy}

Fourier transform infrared spectroscopy (FT-IR) was used to investigate the functional groups in the synthesized materials. The results are shown in Figure 3. The spectra of all modified SBA-15 materials exhibited the following bands: broad O-H stretching at $3440 \mathrm{~cm}^{-1}$; Si-OH bending vibration at $962 \mathrm{~cm}^{-1}$; asymmetric and symmetric stretchings of Si-O-Si at 1084 and $800 \mathrm{~cm}^{-1}$, respectively; and bending vibration of Si-O-Si at $467 \mathrm{~cm}^{-1}$. Furthermore, all modified samples exhibited IR band due to water at $1632 \mathrm{~cm}^{-1}$, which, together with the presence of $\mathrm{Si}-\mathrm{OH}$ band $\left(3440 \mathrm{~cm}^{-1}\right)$, suggests the existence of hydrogen bonding between water molecules and the hydrophilic $\mathrm{Si}-\mathrm{OH}$ groups [19].

FT-IR results also confirm the successful anchoring of HPW on the surface of SBA-15 and its amino-modified analogs. In particular, the peak due to P-O asymmetric stretching of pure HPW solid was observed at $1082 \mathrm{~cm}^{-1}$ and the characteristic peaks were found at 984,893 , and $810 \mathrm{~cm}^{-1}$, which could be designated to the stretching vibrations of terminal asymmetric oxygen $\left(\mathrm{W}=\mathrm{O}_{\mathrm{d}}\right)$, 
corner shared asymmetric oxygen $\left(\mathrm{W}-\mathrm{O}_{\mathrm{b}}-\mathrm{W}\right)$, and edge shared oxygen $\left(\mathrm{W}-\mathrm{O}_{\mathrm{c}}-\mathrm{W}\right)$, respectively [19]. For the modified materials, peaks due to $\mathrm{W}=\mathrm{O}_{\mathrm{d}}$ and $\mathrm{W}-\mathrm{O}_{\mathrm{b}}-\mathrm{W}$ stretching vibrations were observed with some red shifts due to strong electrostatic interactions between the electron-rich HPW anions and the protonated amino-functionalized SBA-15 material. For instance, the stretching vibrations of $\mathrm{W}=\mathrm{O}_{\mathrm{d}}$ and $\mathrm{W}-\mathrm{O}_{\mathrm{b}}-\mathrm{W}$ in S-N $\mathrm{N}^{1}-\mathrm{HPW}$ were observed at 943 and $881 \mathrm{~cm}^{-1}$, respectively.

The successful protonation of the amino groups by HPW was also confirmed by the FT-IR results. Specifically, peaks that can be attributed to $-\mathrm{NH}_{3}{ }^{+}$and $-\mathrm{NH}_{2}{ }^{+}$- bending vibrations were observed in the region of $1468-1506 \mathrm{~cm}^{-1}$ in all S-N ${ }^{\mathrm{x}}-\mathrm{HPW}$ samples [22]. These peaks are otherwise absent in the spectrum of S-HPW sample, which is to be expected since this sample did not contain protonated amino groups.

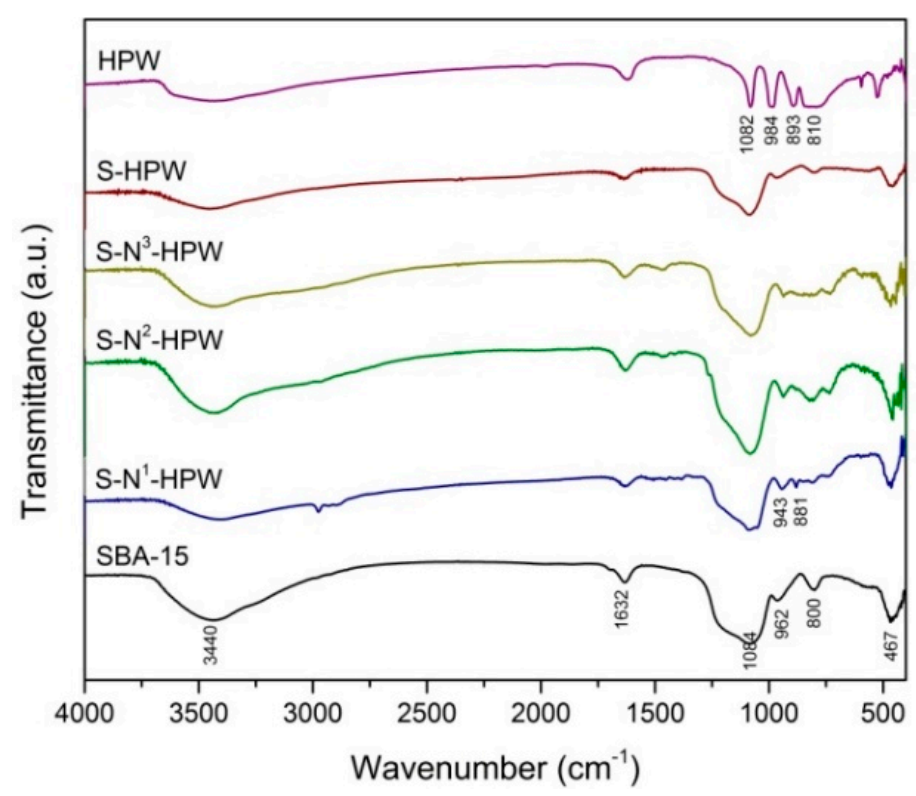

Figure 3. Fourier transform infrared spectroscopy (FT-IR) spectra of SBA-15, HPW, S-N ${ }^{\mathrm{x}}-\mathrm{HPW}$, and S-HPW materials.

\subsubsection{Morphological Analysis}

Morphologies of the representative synthesized materials were examined using scanning electron microscopy (SEM) and transmission electron microscopy (TEM). The results are shown in Figure 4. The SEM images suggest that the rope-like structure of the parent SBA-15 (Figure 4a) was preserved after modification with aminosilane and HPW to produce S-N $\mathrm{N}^{\mathrm{x}}-\mathrm{HPW}$ (Figure $4 \mathrm{~b}-\mathrm{d}$ ). The SEM images of S-N ${ }^{\mathrm{x}}-\mathrm{HPW}$ also reveal that functionalizing SBA-15 with aminosilanes prior to modification with HPW facilitated the distribution of the latter via the strong interaction of HPW anions and the protonated amino groups. In particular, no large agglomeration of HPW nanoparticles was observed on the external surface of the support. The presence of aminosilane, however, does not necessarily prevent the interaction between terminal oxygen atoms of HPW and the silanol hydroxyls of the SBA-15 support [27].

SEM-EDX was used to further study the distribution of HPW nanoparticles on the support. The results (Table 2) suggest that HPW nanoparticles were substantially uniformly distributed throughout the surface of the amino-functionalized SBA-15, but that the distribution was not uniform on the non-aminosilylated support. In particular, the weight ratios of $\mathrm{Si} / \mathrm{W}$ measured at three randomly selected regions of each S-N $\mathrm{N}^{\mathrm{x}}$-HPW sample showed a high level of similarity, while those of the S-HPW sample exhibited a large variation. The uniformity of the nanoparticle distribution is likely driven by the strong chemical bonding interaction between the HPW anions and the protonated amino-functionalized support [23]. 
TEM was used to investigate the pore structure of the materials. The results for the representative synthesized materials: SBA-15 and S-N²-HPW (Figure 4e,f) indicate that the ordered 2D mesoporous structure of SBA-15 was retained after the amino-functionalization and acid immobilization. This finding is in good agreement with the above $\mathrm{XRD}, \mathrm{N}_{2}$ adsorption-desorption, and FT-IR results. Apart from SEM and TEM observations, the fact that no yellow color was observed in the synthesized materials indicates that decomposition of $\mathrm{HPW}$ to $\mathrm{WO}_{3}$ did not occur during the synthesis. Overall, the fact that aminosilane grafting and HPW immobilization did not destroy the skeleton structure of SBA-15 demonstrates that the latter is a suitable support material for the purpose of this study.

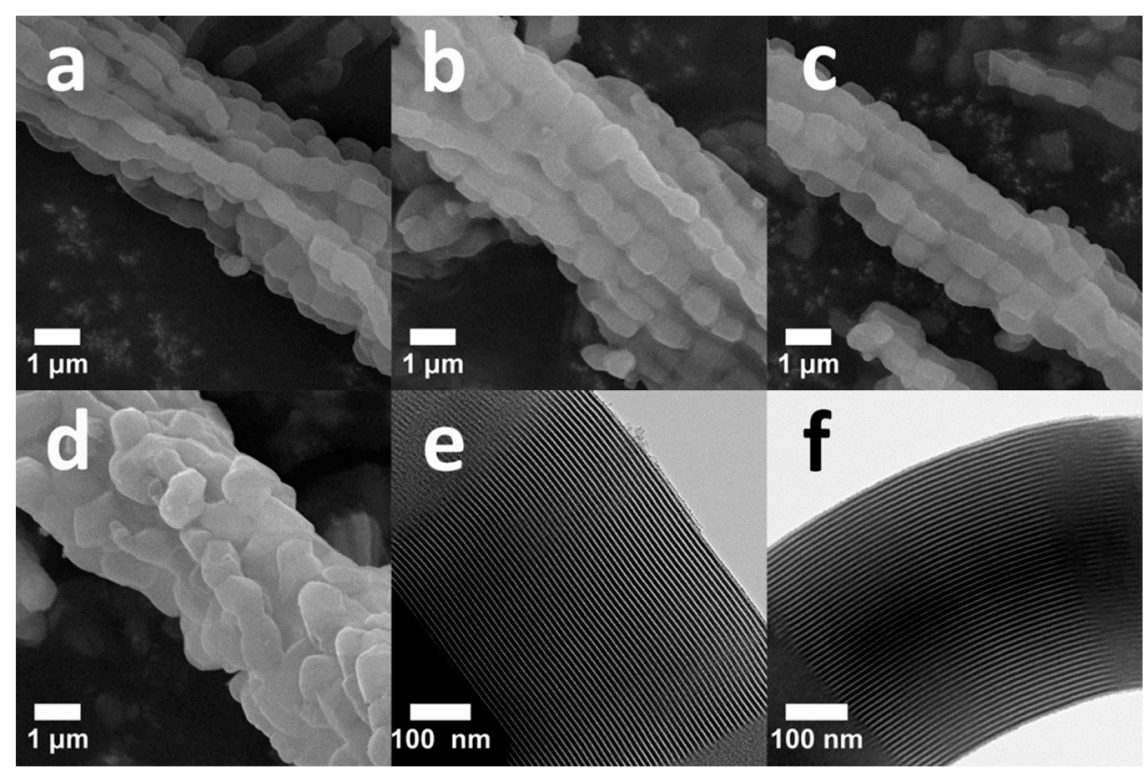

Figure 4. Scanning electron microscopy (SEM) images of SBA-15 (a), S- $\mathrm{N}^{1}-\mathrm{HPW}(\mathbf{b}), \mathrm{S}^{-\mathrm{N}^{2}-\mathrm{HPW}}(\mathbf{c})$, and S-N ${ }^{3}-\mathrm{HPW}(\mathbf{d})$; transmission electron microscopy (TEM) images of SBA-15 (e) and S-N $\mathrm{N}^{2}-\mathrm{HPW}(\mathbf{f})$.

Table 2. Weight ratios of Si/W in S-N ${ }^{\mathrm{x}}-\mathrm{HPW}$ and S-HPW materials measured by SEM equipped with energy dispersive $\mathrm{X}$-ray spectroscopy (SEM-EDX) at three randomly selected regions.

\begin{tabular}{ccccc}
\hline \multirow{2}{*}{ Materials } & \multicolumn{4}{c}{ Weight Ratios of Si/W } \\
\cline { 2 - 5 } & Region 1 & Region 2 & Region 3 & S.D. \\
\hline S-N ${ }^{1}$-HPW & 0.871 & 0.873 & 0.829 & 0.025 \\
S-N ${ }^{2}$-HPW & 0.769 & 0.768 & 0.770 & 0.001 \\
S-N ${ }^{3}$-HPW & 0.611 & 0.609 & 0.612 & 0.002 \\
S-HPW & 8.522 & 8.173 & 15.486 & 4.125 \\
\hline
\end{tabular}

\subsubsection{Elemental Analysis and Acidity}

The elemental analyzer was used to determine the nitrogen content of the synthesized materials. The results, as shown in Table 1, suggest that the aminosilylation on the surface of SBA-15 was successful. In particular, the nitrogen content in S-N $\mathrm{N}^{1} \mathrm{HPW}\left(1.34\right.$ mmole $\left.^{-1}\right), \mathrm{S}^{-\mathrm{N}^{2}-\mathrm{HPW}}\left(1.78 \mathrm{mmole}^{-1}\right)$, and S- $\mathrm{N}^{3}-\mathrm{HPW}\left(2.87 \mathrm{mmole}^{-1}\right)$ increased with increasing number of amino groups in the aminosilanes, and nitrogen was not detected in pure SBA-15 and S-HPW.

Greater presence of amino groups was found to affect the acidity of the materials (Table 1). Specifically, the Brønsted acidity of the S-N $\mathrm{N}^{\mathrm{x}}-\mathrm{HPW}$ materials increased with increasing presence of amino groups: $0.32,0.41$, and 0.47 mmole $^{-1}$ for S- $\mathrm{N}^{1}-\mathrm{HPW}, \mathrm{S}-\mathrm{N}^{2}-\mathrm{HPW}$, and S- $\mathrm{N}^{3}-\mathrm{HPW}$, respectively. The tungsten content found in the S-N $\mathrm{N}^{\mathrm{x}}$-HPW materials, which reflects HPW loading, well corresponds to the trend of acidity in Table 1 . This finding is likely due to the fact that larger amount of amino groups led to greater availability of protonation sites. In the absence of amino group, however, 
S-HPW also showed a relatively high Brønsted acidity $\left(0.31 \mathrm{mmole}^{-1}\right)$. This latter finding could be attributed to the fact that the terminal oxygens of HPW can form hydrogen bonding with the surface silanol groups of SBA-15 [31], and that these silanol groups may also be protonated by HPW to result in an interaction between the positively charged silica species and HPW anions [26].

\subsection{Catalytic Performance of the Synthesized Catalysts}

The modified SBA-15 catalysts were tested for their catalytic performance in the esterification of glycerol with oleic acid under the following conditions: $10-300 \mathrm{~min}, 160^{\circ} \mathrm{C}$, glycerol/oleic acid molar ratio of 4:1, and $2.5 \mathrm{wt} \%$ of catalyst loading. The results are shown in Figure 5. The conversion of oleic acid (Figure 5a), representative of the catalyst activity, increased rapidly for all catalysts during the first 60 min. Specifically, S-N ${ }^{1}-\mathrm{HPW}, \mathrm{S}^{-\mathrm{N}^{2}-\mathrm{HPW}}$, S-N ${ }^{3}-\mathrm{HPW}$, and S-HPW catalysts, respectively showed the following rates of conversion during this initial reaction period: 12.8, 17.8, 17.4, and 10.3 mmole $\mathrm{min}^{-1}$. After the first hour of reaction, all catalysts showed progressively slower conversion and, after $5 \mathrm{~h}$ of reaction, the maximum conversions of $82.8 \%, 95.0 \%, 95.6 \%$, and $81.9 \%$ were achieved by S-N ${ }^{1}-\mathrm{HPW}$, S-N ${ }^{2}-\mathrm{HPW}, \mathrm{S}-\mathrm{N}^{3}-\mathrm{HPW}$, and S-HPW, respectively.

During the reaction period, it can be observed that S-N ${ }^{2}-\mathrm{HPW}$ exhibited the highest catalytic activity at nearly all experimental time intervals, followed by the activity exhibited by S-N ${ }^{3}-\mathrm{HPW}$, S-N ${ }^{1}-\mathrm{HPW}$, and S-HPW, respectively. This finding suggests that acidity of the four catalysts was not the only determining factor for the catalytic activity, specifically since the Brønsted acidity of the materials is in the order of S-N $\mathrm{N}^{3}-\mathrm{HPW}>\mathrm{S}-\mathrm{N}^{2}-\mathrm{HPW}>\mathrm{S}-\mathrm{N}^{1}-\mathrm{HPW}>\mathrm{S}-\mathrm{HPW}$ (Table 1). Further analysis revealed that the catalytic activity was also affected by the surface area and pore volume of the materials. In particular, the fact that $\mathrm{S}-\mathrm{N}^{3}-\mathrm{HPW}$ had the smallest BET surface area and pore volume as well as the highest acidity (Table 1) suggests a relatively high occurrence of multilayer formation of acid sites in this catalyst. Such an occurrence can cause a considerable number of the active sites to be blocked and hence decrease the access of the reactants to the active sites [19]. The lower activity observed for $\mathrm{S}-\mathrm{N}^{3}-\mathrm{HPW}$ is therefore an expected consequence.

As for selectivity of the desired monoolein product, it was found that S- $\mathrm{N}^{2}-\mathrm{HPW}$ showed highest selectivity at nearly all conversion levels (e.g., $\sim 78 \%$ at $70 \%$ of conversion), followed by S-N ${ }^{3}-\mathrm{HPW}$ (e.g., $\sim 65 \%$ at $70 \%$ of conversion), S-N ${ }^{1}$-HPW (e.g., $\sim 64 \%$ at $70 \%$ of conversion), and S-HPW (e.g., $\sim 40 \%$ at $70 \%$ conversion) (Figure $5 \mathrm{~b}$ ). It is also to be noted that the selectivity of monoolein decreased with increasing conversion of oleic acid, which occurred due to the further esterification of monoolein with oleic acid to form diolein and triolein (Scheme 1).

Since the levels of monoolein selectivity exhibited by the modified SBA-15 materials do not completely coincide with their relative acidity, pore size, and pore volume, it is quite evident that the differences in selectivity were not dominated by only one of these factors. For the two best catalysts in terms of activity: S-N ${ }^{2}-\mathrm{HPW}$ and S- $\mathrm{N}^{3}-\mathrm{HPW}$, the higher acidity of the latter did not result in superior selectivity much likely due to its low pore volume and small pore size. As discussed above, its high acidity and low pore volume could cause substantial multilayer formation of acid sites. Together with the fact that its pore diameter is small, the accessibility of the reactants to the mesoporous channels decreased. Consequently, the esterification reaction substantially took place on the external surface of $\mathrm{S}-\mathrm{N}^{3}-\mathrm{HPW}$, which is less selective for monoolein as compared to esterification inside the pores.

For the two best catalysts in terms of selectivity: S-N $-\mathrm{HPW}$ and $\mathrm{S}-\mathrm{N}^{2}-\mathrm{HPW}$, the fact that the former exhibited lower selectivity than the latter despite their similar pore characteristics could be attributed to the difference in the availability of acidic active sites. In particular, the relative scarcity of acid sites in the mesoporous channels of S-N $\mathrm{N}^{1}-\mathrm{HPW}$ hindered the occurrence of S-N $\mathrm{N}^{1}-\mathrm{HPW}$-catalyzed esterification inside the pores. The occurrence of autocatalysis by oleic acid therefore proportionately increased. Fundamentally, the autocatalysis could occur since oleic acid can act as a catalyst for its own esterification with glycerol [32,33]. The lower number of active sites in S-N $\mathrm{N}^{1}-\mathrm{HPW}$, therefore, contributed to its inferior selectivity towards monoolein. 
For the non-aminosilylated S-HPW, its monoolein selectivity was found to be lower than that achieved by its aminosilylated counterpart that has similar concentration of acidic active sites: $\mathrm{S}-\mathrm{N}^{1}$-HPW. This finding indicates that lower monoolein selectivity was not only associated with lower pore volume and smaller pore size as in the case of S-N $\mathrm{N}^{3}-\mathrm{HPW}$, but also associated with higher pore volume and larger pore size as in this case. Specifically, it is apparent that the larger pores of S-HPW (i.e., pore diameter in the range of $7.1-16.0 \mathrm{~nm}$ for S-HPW vs. $4.8-8.1 \mathrm{~nm}$ for S-N ${ }^{1}-\mathrm{HPW}$ ) increased the probability of formation of diolein and triolein within the pores through subsequent esterification of monoolein.

Overall, analysis of the catalytic performance indicates that physical and chemical properties of the tested catalysts, especially including the total BET surface area, pore size, pore volume, and acidity of the materials, had synergistic effects on the activity and selectivity of the catalyzed esterification of glycerol with oleic acid to produce monoolein. Due to the higher efficiency in catalyzing the esterification of glycerol with oleic acid achieved by S-N ${ }^{2}-\mathrm{HPW}$, it was chosen for further analysis in this study.
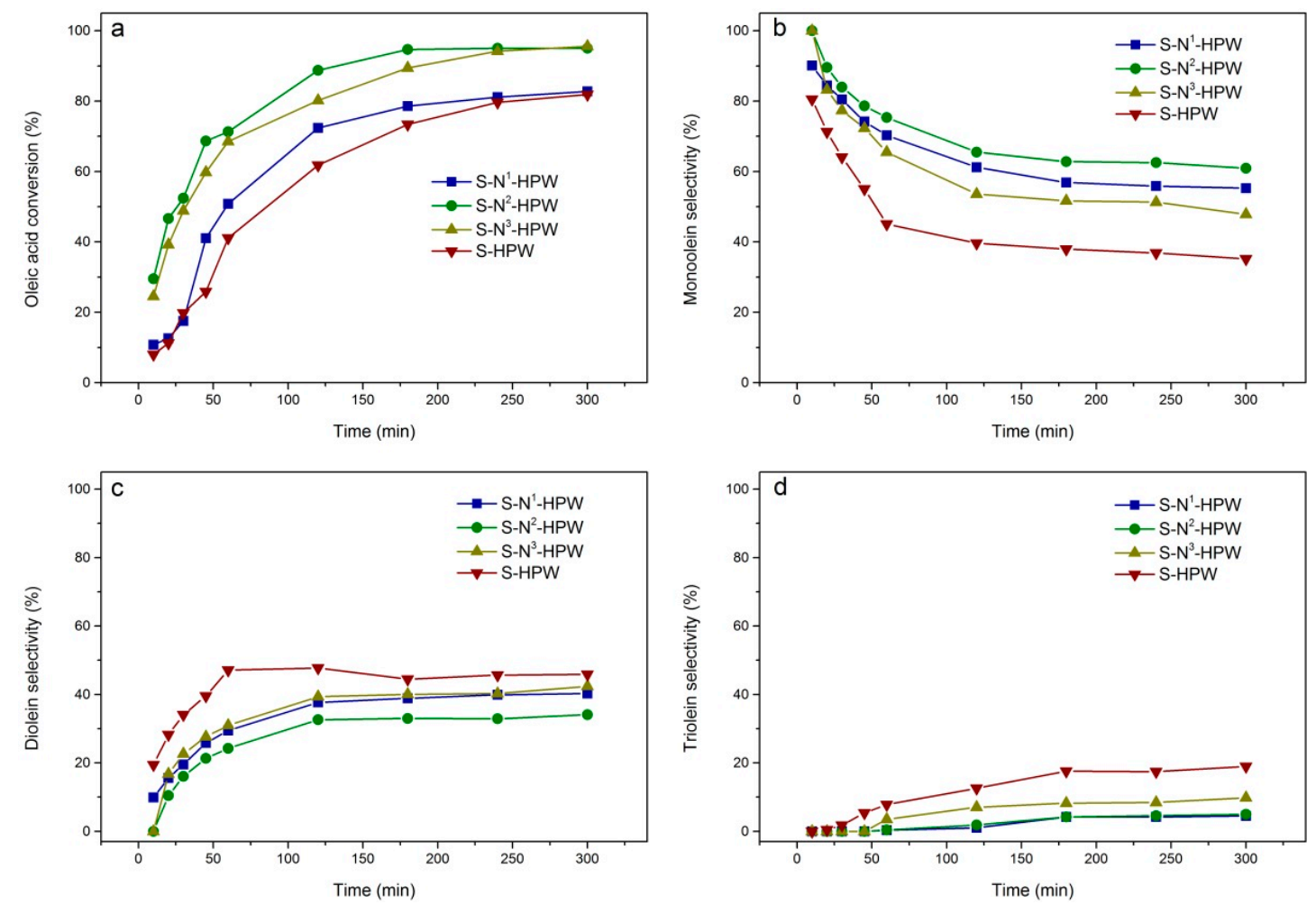

Figure 5. Catalytic activity of S-N $\mathrm{N}^{\mathrm{x}}-\mathrm{HPW}$ and S-HPW catalysts (a) and the corresponding product distributions (b-d) in the esterification of glycerol with oleic acid for $10-300 \mathrm{~min}$ at $160{ }^{\circ} \mathrm{C}$ using glycerol/oleic acid molar ratio of $4: 1$ and $2.5 \mathrm{wt} \%$ of catalyst loading.

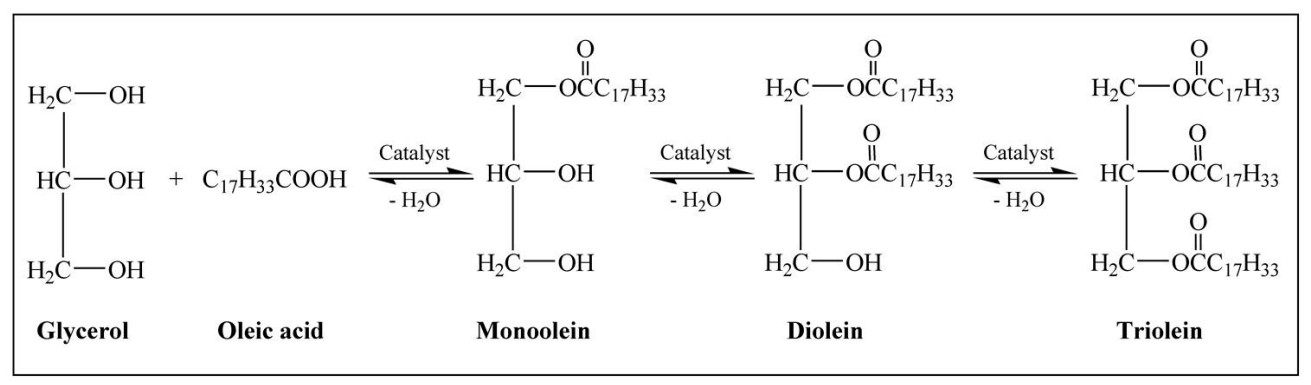

Scheme 1. Esterification of glycerol with oleic acid to produce olein products (isomers are not shown). 


\subsection{Effect of Reaction Parameters}

This section investigates the effect of various reaction parameters of the esterification of glycerol with oleic acid, including temperature, initial ratio of starting materials, and catalyst loading, on the conversion of oleic acid and the selectivity of glyceride products using S- $\mathrm{N}^{2}-\mathrm{HPW}$ as a representative catalyst. The investigation is important particularly because conversion and product distribution of catalyzed esterification of glycerol and fatty acids may not only be dependent on the nature of the catalyst but may also be affected by reaction parameters $[2,19,33,34]$.

\subsubsection{Effect of Reaction Temperature}

The effect of reaction temperature on the conversion of oleic acid and selectivity of glyceride products is shown in Figure 6. The esterification was performed using the S- $\mathrm{N}^{2}-\mathrm{HPW}$ catalyst for $3 \mathrm{~h}$ with glycerol/oleic acid molar ratio of $4: 1,2.5 \mathrm{wt} \%$ of catalyst loading, and reaction temperature ranging from 120 to $180^{\circ} \mathrm{C}$. The results indicate that the reaction temperature had a significant influence on both the conversion and selectivity. In particular, a significant increase in oleic acid conversion was observed with increasing temperature from 120 to $160^{\circ} \mathrm{C}$. This finding suggests that the increase in temperature improved the effectiveness of the collision between molecules of glycerol and oleic acid reactants, which in turn increased the probability that the kinetic energy of the reactant molecules exceeded the activation energy required for successful conversion [35].

Increasing the reaction temperature from 120 to $160^{\circ} \mathrm{C}$ was, on the other hand, associated with a decrease in monoolein selectivity and with an increase in selectivities of the undesired diolein and triolein. This occurrence suggests that the higher reaction temperature increased kinetic energy of the reactant molecules sufficiently to overcome the higher activation energy barriers for the formation of the bulkier diolein and triolein molecules. The effect of reaction temperature is, however, not linear. Specifically, further increasing the temperature from 160 to $180{ }^{\circ} \mathrm{C}$ was associated with relatively small changes in the conversion and product selectivity. This latter finding suggests that, at this high temperature level, the reaction rate was sufficiently fast that the equilibrium was reached before $3 \mathrm{~h} \mathrm{[12].}$

It is to be noted that although a higher conversion of oleic acid was obtained by increasing the reaction temperature, too high temperature may lead to undesired outcomes such as change in color of the glyceride products. In particular, it can be observed (Figure 6) that the color of the reaction mixture became significantly darker when the reaction temperature was increased from 160 to $180{ }^{\circ} \mathrm{C}$ as compared to the color observed at lower temperature levels. This finding is likely attributable to the degradation of oleic acid and the glyceride products $[34,35]$ that occurred predominantly at very high reaction temperatures.

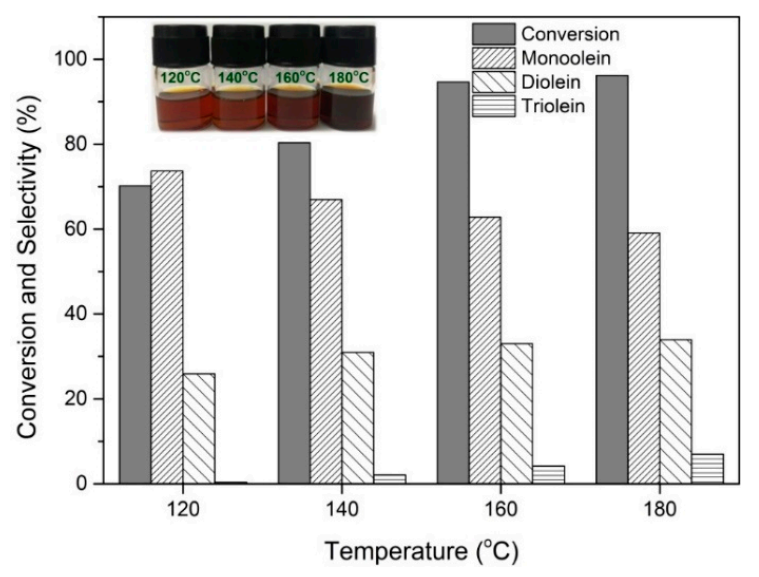

Figure 6. Effect of reaction temperature on oleic acid conversion, product distribution, and product color using S-N ${ }^{2}$-HPW catalyst in esterification of glycerol with oleic acid for $3 \mathrm{~h}$ using glycerol/oleic acid molar ratio of $4: 1,2.5 \mathrm{wt} \%$ of catalyst loading, and reaction temperatures of $120,140,160$, and $180{ }^{\circ} \mathrm{C}$. 
As a result, it can be concluded that optimization of the reaction temperature is critical for maximizing the yield of monoolein. Of no less importance, the balance between gain in conversion and loss in selectivity needs to be taken into account for the calibration of temperature. Also, since the physical properties of the product such as color may be affected by changes in reaction temperature, this factor must also be considered in the optimization process in order to maintain the quality of the product.

\subsubsection{Effect of Catalyst Loading}

The effect of catalyst loading on the conversion of oleic acid and selectivity of glyceride products is shown in Figure 7. The esterification was performed using the S-N ${ }^{2}-\mathrm{HPW}$ catalyst at $160{ }^{\circ} \mathrm{C}$ for $3 \mathrm{~h}$ with glycerol/oleic acid molar ratio of 4:1 and catalyst loadings of 1.5, 2.5, and $3.5 \mathrm{wt} \%$. The results indicate that catalyst loading had a significant effect on the conversion of oleic acid. In particular, increasing the catalyst loading from 1.5 to $2.5 \mathrm{wt} \%$ was associated with a large increase in oleic acid conversion from $64 \%$ to $95 \%$. This finding is likely attributable to the enhanced accessibility of acidic sites. Product distribution, on the other hand, was minimally affected by this change in catalyst loading, indicating that the enhanced conversion was also accompanied by a greater presence of further esterification of monoolein with oleic acid to form diolein and triolein. Further increasing the catalyst loading from 2.5 to $3.5 \mathrm{wt} \%$ was associated with no improvement in oleic acid conversion and no significant change in product distribution. This latter observation is likely due to the increment of acidic sites being in excess of that required by the starting materials to react under the present conditions [35].

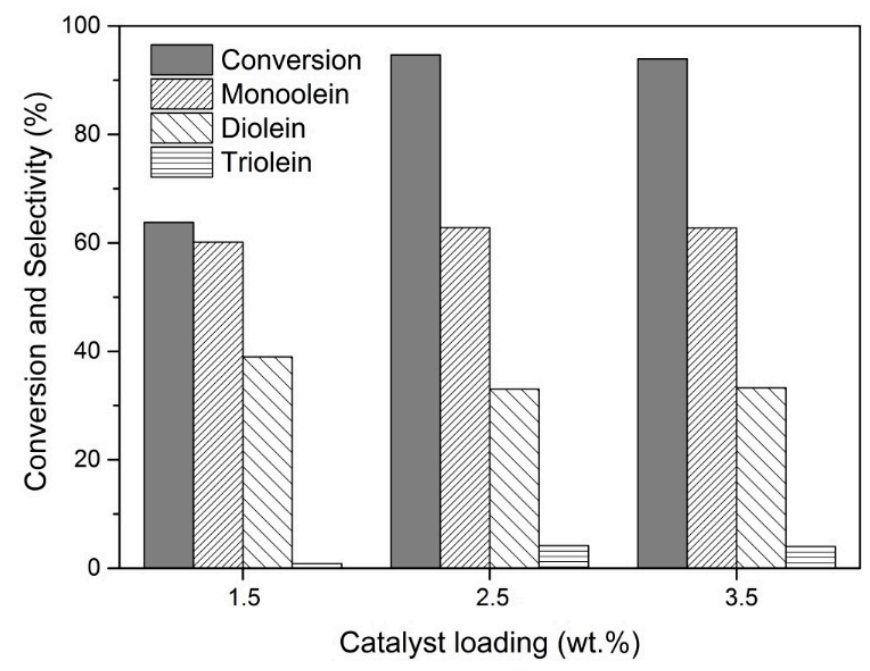

Figure 7. Effect of catalyst loading on oleic acid conversion and product distribution using S-N ${ }^{2}-\mathrm{HPW}$ catalyst in esterification of glycerol with oleic acid at $160{ }^{\circ} \mathrm{C}$ for $3 \mathrm{~h}$ with glycerol/oleic acid molar ratio of $4: 1$ and catalyst loadings of $1.5,2.5$, and $3.5 \mathrm{wt} \%$.

\subsubsection{Effect of Glycerol/Oleic Acid Molar Ratio}

The effect of glycerol/oleic acid molar ratio on the conversion of oleic acid and selectivity of glyceride products is shown in Figure 8. The esterification was performed using the S-N ${ }^{2}-\mathrm{HPW}$ catalyst at $160{ }^{\circ} \mathrm{C}$ for $3 \mathrm{~h}$ with $2.5 \mathrm{wt} \%$ of catalyst loading and glycerol/oleic acid molar ratios of 1:1, 2:1, 4:1, and 6:1. The results indicate that glycerol/oleic acid molar ratio significantly affected both the conversion and the selectivity. For oleic acid conversion, increasing the molar ratio of glycerol/oleic acid from 1:1 to $4: 1$ resulted in an increase in the conversion from $86 \%$ to $95 \%$. This finding is in line with Le Chatelier's principle and the fact that the esterification reaction of glycerol with oleic acid is equilibrium-limited. Specifically, an increase in the concentration of either glycerol or oleic 
acid is expected to drive the reaction forward and raise the conversion of oleic acid. Nevertheless, further increasing the molar ratio to $6: 1$ resulted in a slight drop in conversion to $91 \%$. The latter occurrence is likely attributable to the retarded formation and diffusion of glyceride products caused by an excess amount of glycerol [36].

For selectivity of glyceride products, increasing the molar ratio of glycerol/oleic acid from 1:1 to $6: 1$ was associated with a large increase in monoglyceride selectivity from $41.8 \%$ to $63.1 \%$. The corresponding decrease in selectivities of both diglyceride and triglyceride was also observed. This finding suggests that increasing glycerol/oleic acid molar ratio enhanced the chances for oleic acid to react with glycerol to form monoolein rather than to react with the generated monoolein and diolein to form diolein and triolein, respectively.

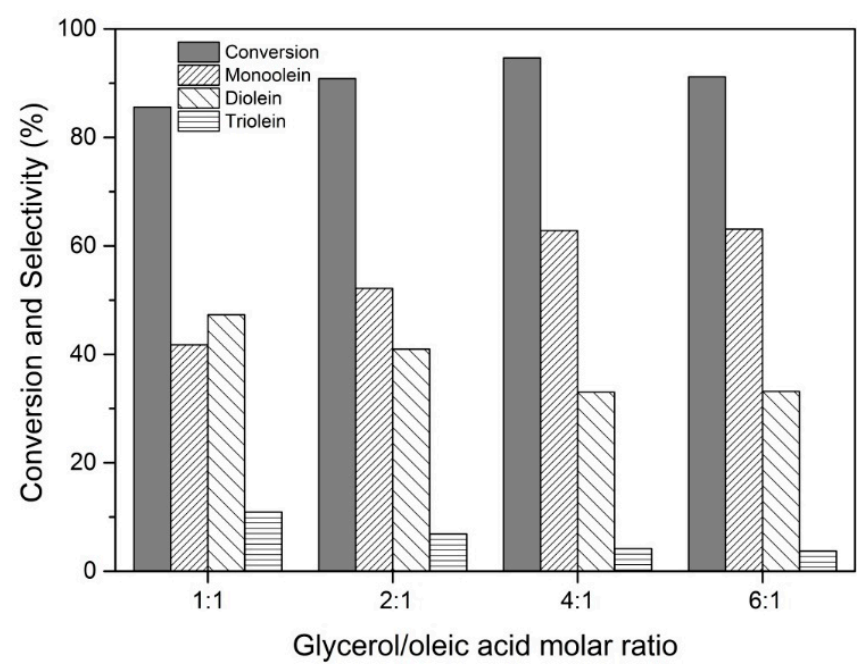

Figure 8. Effect of glycerol/oleic acid molar ratio on oleic acid conversion and product distribution using S-N ${ }^{2}$-HPW catalyst in esterification of glycerol with oleic acid at $160{ }^{\circ} \mathrm{C}$ for $3 \mathrm{~h}$ with $2.5 \mathrm{wt} \%$ of catalyst loading and glycerol/oleic acid molar ratios of 1:1, 2:1, 4:1, and 6:1.

\subsection{Reusability of the Catalysts}

This section investigates the effect of functionalization of SBA- 15 with aminosilane prior to the immobilization of HPW on the reusability of the materials when used as catalysts for the esterification of glycerol with oleic acid. The esterification was performed for six consecutive cycles at $160{ }^{\circ} \mathrm{C}$ with $2.5 \mathrm{wt} \%$ of catalyst loading and glycerol/oleic acid molar ratios of 4:1 for $3 \mathrm{~h}$ per cycle. The S-HPW and $\mathrm{S}-\mathrm{N}^{2}-\mathrm{HPW}$ were used as representative catalysts. The result indicates that aminosilylation improved catalyst reusability. As shown in Figure 9, the loss in catalytic activity was much less pronounced in the case of S-N $\mathrm{N}^{2}-\mathrm{HPW}$ as compared to S-HPW. In particular, the conversion of oleic acid over S-N ${ }^{2}-\mathrm{HPW}$ decreased from $91.4 \%$ in the first cycle to $84.3 \%$ in the sixth cycle, constituting a $7.8 \%$ decrease, while the conversion over S-HPW decreased from $71.5 \%$ in the first cycle to $56.0 \%$ in the sixth cycle, constituting a $21.7 \%$ decrease. Both catalysts, however, showed no significant loss in monoolein selectivity over the six cycles of reuse (Figure S1).

The higher reusability of S-N ${ }^{2}-\mathrm{HPW}$ can be attributed to the enhanced stability provided by amino functionalization. Specifically, the ionic bonding between the protonated amino groups and HPW anions in S- $\mathrm{N}^{2}-\mathrm{HPW}$ is much stronger than the hydrogen bonding between terminal oxygens of HPW and the surface silanol groups of SBA-15 in S-HPW. The stronger bonding in S-N ${ }^{2}-\mathrm{HPW}$ was also followed by less acid-site leaching effects. In particular, ICP-OES results indicate that the content of tungsten leached into the reaction mixture containing the S- $\mathrm{N}^{2}-\mathrm{HPW}$ catalyst and that containing the S-HPW catalyst after the third cycle of reaction was 0.06 and $0.44 \mathrm{wt} \%$, respectively. Good reusability of HPW-incorporated mesoporous silica-based heterogeneous catalysts that involve 
the functionalization of amino groups was also observed in other reactions such as acidolysis of soybean oil [22], epoxidation of dicyclopentadiene [23], oxidation of benzyl alcohol [29], and acetylation of anisole [37].

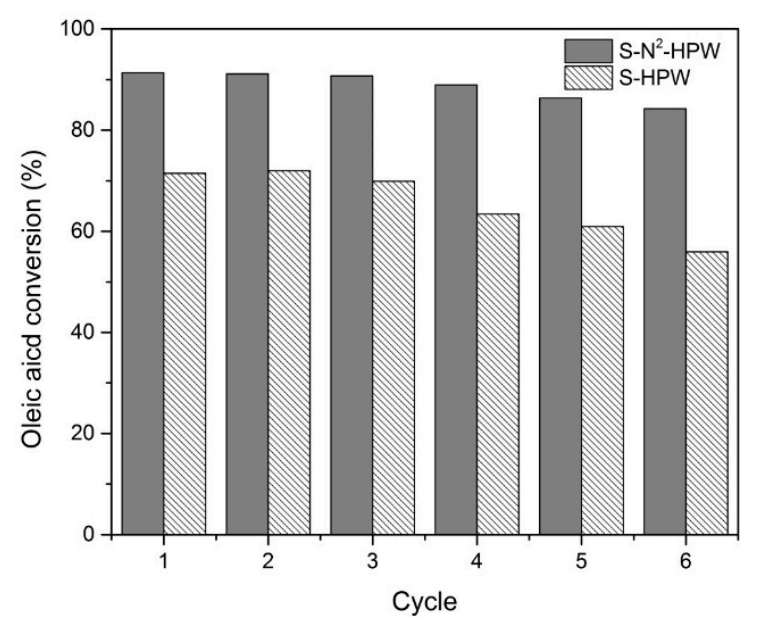

Figure 9. Effect of aminosilane functionalization on catalyst reusability using S- $\mathrm{N}^{2}-\mathrm{HPW}$ and S-HPW catalysts in esterification of glycerol with oleic acid for six cycles at $160^{\circ} \mathrm{C}$ for $3 \mathrm{~h}$ per cycle with $2.5 \mathrm{wt} \%$ of catalyst loading and glycerol/oleic acid molar ratio of 4:1.

\subsection{Comparison of Catalytic Performance}

The catalytic performance in the esterification of glycerol with oleic acid achieved by the most efficient catalyst in the present study, S-N ${ }^{2}-\mathrm{HPW}$, was compared to that of three commercial acid catalysts: HZSM-5, H-BEA, and Amberlyst-15. The esterification was performed at $160{ }^{\circ} \mathrm{C}$ for $3 \mathrm{~h}$ with $2.5 \mathrm{wt} \%$ of catalyst loading and glycerol/oleic acid molar ratio of 4:1. The results (Figure 10) indicate that S-N ${ }^{2}$-HPW exhibited higher oleic acid conversion and monoglyceride selectivity $(91.4 \%$ and $64.5 \%$, respectively) as compared to HZSM- 5 (70.8\% and $44.6 \%$, respectively), H-BEA ( $74.0 \%$ and $44.7 \%$, respectively), and Amberlyst- 15 (50.4\% and 39.3\%, respectively).

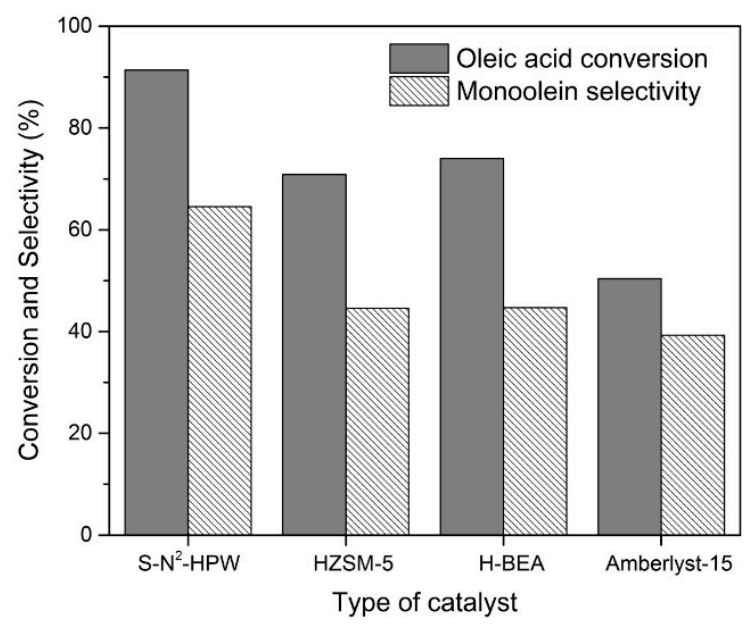

Figure 10. Comparison of catalytic performance of S- $\mathrm{N}^{2}-\mathrm{HPW}$ and three commercial acid catalysts: HZSM-5, H-BEA, and Amberlyst-15 for esterification of glycerol with oleic acid at $160^{\circ} \mathrm{C}$ for $3 \mathrm{~h}$ using glycerol/oleic acid molar ratio of 4:1 and $2.5 \mathrm{wt} \%$ of catalyst loading.

The superiority can be attributed to the better morphology of the S-N2-HPW catalyst. As compared to HZSM-5 and H-BEA, the size of the mesopores of S-N ${ }^{2}-\mathrm{HPW}(7.05 \mathrm{~nm})$ is more favorable 
for the esterification reaction than that of the micropores of these zeolites $(0.63 \mathrm{~nm}$ and $0.66 \mathrm{~nm}$, respectively). Specifically, microporosity can hinder the formation and diffusion of the desired monoolein product [36]. This hindrance can result in the esterification reaction taking place at the external surface of the zeolites, where the availability of active sites is lower than the internal surface of the pores. As for the case of Amberlyst-15, its low BET surface area is among the major causes for the comparatively low catalytic performance, since the accessibility of the acid sites is reduced by this limitation [2].

\section{Materials and Methods}

\subsection{Materials}

Tetraethyl orthosilicate (TEOS), poly(ethylene glycol)-block-poly(propylene glycol)-blockpoly(ethylene glycol) (Pluronic P123, MW 5800), 3-aminopropyltriethoxysilane (APTES), (3-trimethoxysilylpropyl)diethylenetriamine (DETTMS), 12-tungstophosphoric acid hydrate (HPW), Amberlyst-15, and glycerol were purchased from Sigma-Aldrich (St. Louis, MO, USA). Oleic acid was purchased from Fluka (Buchs, Switzerland). [3-(2-Amino-ethylamino)propyltrimethoxysilane] (AAPTMS) was purchased from Alfa Aesar (Ward Hill, MA, USA). HZSM-5 (Si/Al = 14.1) and H-BEA $(\mathrm{Si} / \mathrm{Al}=15)$ were purchased from ZEOCHEM (Zürich, Switzerland). All chemicals were analytical grade reagents and were used without further purification.

\subsection{Synthesis of Amino-Functionalized SBA-15 Materials}

The SBA-15 mesoporous material with an ordered structure was hydrothermally prepared according to the published procedure [38], which involves the use of triblock co-polymer Pluronic P123 as the surfactant template under acidic conditions. Amino-functionalized SBA-15 materials were prepared by post-grafting method modified from a procedure described in the literature [39]. Three types of aminosilanes: APTES, AAPTMS, and DETTMS, whose structures are shown in Scheme 2, were used to functionalize the SBA-15 material. Briefly, the functionalization was performed as follows: $1 \mathrm{~g}$ of the synthesized SBA-15 was refluxed in $50 \mathrm{~mL}$ of anhydrous toluene. Then, $1 \mathrm{~mL}$ of each type of aminosilane was added dropwise to each of the resulting mixtures. The reaction was further refluxed for $24 \mathrm{~h}$ under nitrogen atmosphere. The resulting solid was separated from each mixture by centrifugation, washed with ethanol, and dried at $60^{\circ} \mathrm{C}$ for $12 \mathrm{~h}$.

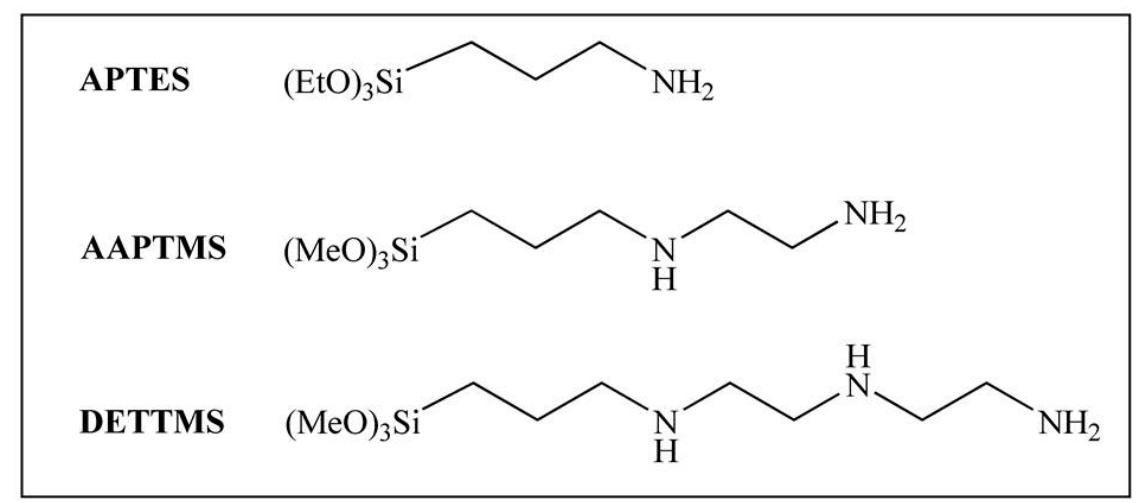

Scheme 2. Chemical structures of aminosilanes used in this work.

\subsection{Synthesis of Protonated Amino-Functionalized SBA-15 Materials}

Protonated amino-functionalized SBA-15 materials were prepared by the impregnation method. The preparation was as follows: $1 \mathrm{~g}$ of each type of amino-functionalized SBA-15 material was added to $120 \mathrm{~mL}$ of deionized water. Then, $0.67 \mathrm{~g}$ of 12 -tungstophosphoric acid (HPW) was added to each of the above mixtures under continuous stirring at room temperature for $24 \mathrm{~h}$. Finally, the resulting 
product was separated from each mixture by centrifugation and washed several times with deionized water and acetone before being dried at $100^{\circ} \mathrm{C}$ for $24 \mathrm{~h}$. The prepared protonated amino-functionalized SBA-15 materials were designated as S- $\mathrm{N}^{\mathrm{x}}-\mathrm{HPW}$, where $\mathrm{x}$ is the number of amino group(s) in the structure of aminosilanes used: $\mathrm{x}=1$ for APTES; $\mathrm{x}=2$ for AAPTMS; and $\mathrm{x}=3$ for DETTMS (Scheme 3 ). For comparison purposes, HPW-impregnated SBA-15 (S-HPW) was also prepared by a procedure similar to that described for the S-Nx $-\mathrm{HPW}$ materials but without the addition of aminosilane.

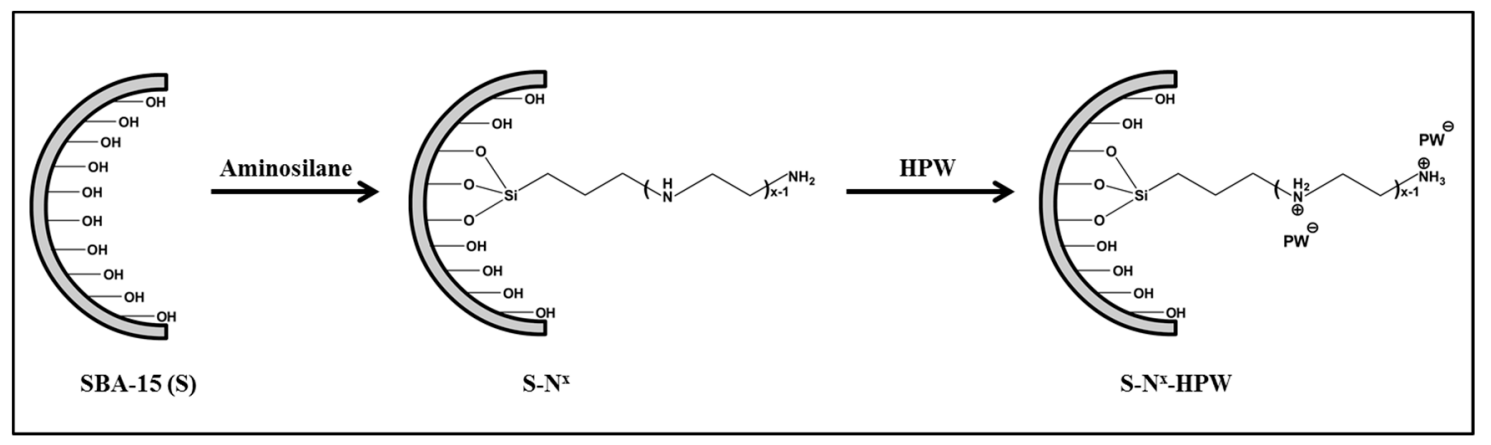

Scheme 3. Synthesis of S-N ${ }^{x}-H P W$ catalysts.

\subsection{Materials Characterization}

The crystallinity of the synthesized materials was investigated by X-ray diffractometer (XRD) (Rigaku D/MaX-2200 Ultima-plus, Tokyo, Japan) with a $\mathrm{Cu} \mathrm{K \alpha}$ X-ray source (1.5418 $\AA$ ) operating at $30 \mathrm{~mA}$ and $40 \mathrm{kV}$. The XRD data were collected in the ranges of $2 \theta=0.7^{\circ}-5^{\circ}$ (low angles) and $2 \theta=15^{\circ}-70^{\circ}$ (high angles) with a scanning rate of $5^{\circ} \mathrm{min}^{-1}$. The textural properties of the materials were examined by nitrogen adsorption-desorption isotherms (BELSORP, mini-II nitrogen adsorptometer, Osaka, Japan). Surface areas were calculated using the Brunauer-Emmett-Teller (BET) method. The total pore volumes and pore sizes were determined using the Barrett-Joyner-Halenda $(\mathrm{BJH})$ method. Functional groups present in the synthesized materials were confirmed by Fourier transform infrared spectrometer (FT-IR) (Nicolet Impact 410, SpectraLab Scientific Inc. Markham, ON, Canada). The morphologies of the materials were investigated by transmission electron microscope (TEM) (JEM-2100, Peabody, MA, USA) and scanning electron microscope (SEM) (JSM-5410 LV, Peabody, MA, USA). The atomic ratios of $\mathrm{Si} / \mathrm{W}$ in the materials were determined by SEM equipped with energy dispersive X-ray spectroscopy (EDX, INCAx-sight, Halifax Road, High Wycombe, UK). The nitrogen content in the materials was analyzed by elemental analyzer (Thermo Scientific FLASH 2000, Waltham, MA, USA). The content of tungsten leached during the reaction was determined by inductively coupled plasma optical emission spectrophotometer (ICP-OES) (Perkin Elmer Optima 2100, Waltham, MA, USA).

\subsection{Catalytic Esterification of Glycerol with Oleic Acid}

The esterification of glycerol with oleic acid was carried out under nitrogen atmosphere in a two-necked round bottom flask equipped with an oil bath, a water-cooled condenser, and a magnetic stirring bar. Unless otherwise stated, the reaction was performed using a 4:1 molar ratio of glycerol to oleic acid and $2.5 \mathrm{wt} \%$ of catalyst at $160^{\circ} \mathrm{C}$. Samples were taken at periodic intervals throughout the $5 \mathrm{~h}$ reaction period where remaining oleic acid and glyceride products were derivatized with $\mathrm{N}$-methyl- $\mathrm{N}$-(trimethylsilyl)trifluoroacetamide and analyzed by gas chromatography (GC) equipped with flame ionization detector (FID) (Varian CP-3800, Palo Alto, CA, USA) using eicosane as the internal standard. All reactions were performed in three replicates and the experimental errors were below $5 \%$. The only products detected in the experiments were mono-, di-, and triolein. The oleic acid conversion and glyceride selectivity were calculated according to methods reported by Hermida et al. [35]. 


\subsection{Catalyst Reusability Test}

After each cycle of the reaction, the catalyst was separated from the reaction mixture by centrifugation at $4000 \mathrm{rpm}$. The separated catalyst was then washed with acetone and cyclohexane, dried at $100{ }^{\circ} \mathrm{C}$ for $24 \mathrm{~h}$, and reused under the same reaction conditions.

\section{Conclusions}

HPW-immobilized SBA-15 (S-HPW) and amino-functionalized HPW-immobilized SBA-15 (S-N $\mathrm{N}^{\mathrm{x}}$-HPW; $\mathrm{N}^{1}, \mathrm{~N}^{2}$, and $\mathrm{N}^{3}$ denote APTES, DETTMS, and AAPTMS, respectively) catalysts were successfully synthesized, where functionalization was accomplished prior to immobilization. The S-N ${ }^{\mathrm{x}}$-HPW catalysts showed superior catalytic performance, as compared to the non-amino functionalized S-HPW catalyst, for glycerol esterification with long-chain oleic acid to produce monoolein. S- $\mathrm{N}^{2}-\mathrm{HPW}$, in particular, showed the highest catalytic performance both in terms of oleic acid conversion and monoolein selectivity, owing to its optimum Brønsted acid density and pore characteristics with respect to the particle size of the desired monoolein product. The synergistic effects of acidity and pore structure helped to minimize pore blockage as well as further esterification to form the bulkier di- and triolein products while maintaining high activity. Aminosilylation was also found to improve catalyst reusability, owing to the strong electrostatic interaction between the protonated amino groups and the HPW anions. Overall, it can be concluded that surface modification using HPW and different types of aminosilanes can be performed to tune the physicochemical properties of catalysts to suit the reaction conditions as well as the physicochemical properties of reactants and products in order to obtain an optimized catalytic performance. These insights can also be applied to the development of better and environmentally friendly catalytic systems for various industrially important reactions.

Supplementary Materials: The following is available online at http://www.mdpi.com/2073-4344/8/9/360/s1, Figure S1: Effect of aminosilane functionalization on catalyst reusability using S- $\mathrm{N}^{2}-\mathrm{HPW}$ and S-HPW catalysts in esterification of glycerol with oleic acid for six cycles at $160^{\circ} \mathrm{C}$ for $3 \mathrm{~h}$ per cycle with $2.5 \mathrm{wt} \%$ of catalyst loading and glycerol/oleic acid molar ratio of 4:1.

Author Contributions: Conceptualization and Methodology, K.R., P.P., and W.A.; Investigation, K.R., C.T., and W.A.; Methodology, K.R., P.P. and W.A.; Writing-Original Draft Preparation, K.R. and W.A.; Writing-Review \& Editing, P.P. and D.N.T.

Funding: This research was funded by the Thailand Research Fund, grant number [MRG5980055], and Grants for Development of New Faculty Staff, Ratchadaphiseksomphot Endowment Fund, Chulalongkorn University.

Conflicts of Interest: The authors declare no conflict of interest.

\section{References}

1. Rahmat, N.; Abdullah, A.Z.; Mohamed, A.R. Recent progress on innovative and potential technologies for glycerol transformation into fuel additives: A critical review. Renew. Sustain. Energy Rev. 2010, 14, 987-1000. [CrossRef]

2. An, S.; Sun, Y.; Song, D.; Zhang, Q.; Guo, Y.; Shang, Q. Arenesulfonic acid-functionalized alkyl-bridged organosilica hollow nanospheres for selective esterification of glycerol with lauric acid to glycerol monoand dilaurate. J. Catal. 2016, 342, 40-54. [CrossRef]

3. Hamerski, F.; Corazza, M.L. Ldh-catalyzed esterification of lauric acid with glycerol in solvent-free system. Appl. Catal. A 2014, 475, 242-248. [CrossRef]

4. Damstrup, M.L.; Jensen, T.; Sparsø, F.V.; Kiil, S.Z.; Jensen, A.D.; Xu, X. Solvent optimization for efficient enzymatic monoacylglycerol production based on a glycerolysis reaction. J. Am. Oil Chem. Soc. 2005, 82, 559-564. [CrossRef]

5. Bornscheuer, U.T. Lipase-catalyzed syntheses of monoacylglycerols. Enzyme Microb. Technol. 1995, 17, 578-586. [CrossRef]

6. Dosuna-Rodríguez, I.; Gaigneaux, E.M. Glycerol acetylation catalysed by ion exchange resins. Catal. Today 2012, 195, 14-21. [CrossRef] 
7. Tangestanifard, M.; Ghaziaskar, H. Arenesulfonic acid-functionalized bentonite as catalyst in glycerol esterification with acetic acid. Catalysts 2017, 7, 211. [CrossRef]

8. Abdullah, A.Z.; Gholami, Z.; Ayoub, M.; Gholami, F. Selective monolaurin synthesis through esterification of glycerol using sulfated zirconia-loaded SBA-15 catalyst. Chem. Eng. Commun. 2015, 203, 496-504. [CrossRef]

9. Wee, L.H.; Lescouet, T.; Fritsch, J.; Bonino, F.; Rose, M.; Sui, Z.; Garrier, E.; Packet, D.; Bordiga, S.; Kaskel, S.; et al. Synthesis of monoglycerides by esterification of oleic acid with glycerol in heterogeneous catalytic process using tin-organic framework catalyst. Catal. Lett. 2013, 143, 356-363. [CrossRef]

10. Machado, M.S.; Pérez-Pariente, J.; Sastre, E.; Cardoso, D.; de Guereñu, A.M. Selective synthesis of glycerol monolaurate with zeolitic molecular sieves. Appl. Catal. A 2000, 203, 321-328. [CrossRef]

11. Corma, A.; Iborra, S.; Miquel, S.; Primo, J. Catalysts for the production of fine chemicals: Production of food emulsifiers, monoglycerides, by glycerolysis of fats with solid base catalysts. J. Catal. 1998, 173, 315-321. [CrossRef]

12. Patel, A.; Singh, S. A green and sustainable approach for esterification of glycerol using 12-tungstophosphoric acid anchored to different supports: Kinetics and effect of support. Fuel 2014, 118, 358-364. [CrossRef]

13. Müller, A.; Peters, F.; Pope, M.T.; Gatteschi, D. Polyoxometalates: Very large clusters-nanoscale magnets. Chem. Rev. 1998, 98, 239-272. [CrossRef] [PubMed]

14. Timofeeva, M.N. Acid catalysis by heteropoly acids. Appl. Catal. A 2003, 256, 19-35. [CrossRef]

15. Misono, M. Unique acid catalysis of heteropoly compounds (heteropolyoxometalates) in the solid state. Chem. Commun. 2001, 1141-1152. [CrossRef]

16. Obalı, Z.; Doğu, T. Activated carbon-tungstophosphoric acid catalysts for the synthesis of tert-amyl ethyl ether (TAEE). Chem. Eng. J. 2008, 138, 548-555. [CrossRef]

17. Jović, A.; Bajuk-Bogdanović, D.; Nedić Vasiljević, B.; Milojević-Rakić, M.; Krajišnik, D.; Dondur, V.; Popa, A.; Uskoković-Marković, S.; Holclajtner-Antunović, I. Synthesis and characterization of 12-phosphotungstic acid supported on BEA zeolite. Mater. Chem. Phys. 2017, 186, 430-437. [CrossRef]

18. Labaki, M.; Mokhtari, M.; Brilhac, J.F.; Thomas, S.; Pitchon, V. Simulation of $\mathrm{NO}$ and $\mathrm{NO}_{2}$ sorption-desorption-reduction behaviours on pt-impregnated hpw supported on $\mathrm{TiO}_{2}$. Appl. Catal. B 2007, 76, 386-394. [CrossRef]

19. Hoo, P.-Y.; Abdullah, A.Z. Direct synthesis of mesoporous 12-tungstophosphoric acid SBA-15 catalyst for selective esterification of glycerol and lauric acid to monolaurate. Chem. Eng. J. 2014, 250, 274-287. [CrossRef]

20. Thielemann, J.P.; Girgsdies, F.; Schlogl, R.; Hess, C. Pore structure and surface area of silica SBA-15: Influence of washing and scale-up. Beilstein J. Nanotechnol. 2011, 2, 110-118. [CrossRef] [PubMed]

21. Dutta, P.; Roy, S.C.; Nandi, L.N.; Samuel, P.; Pillai, S.M.; Bhat, B.D.; Ravindranathan, M. Synthesis of lower olefins from methanol and subsequent conversion of ethylene to higher olefins via oligomerisation. J. Mol. Catal. A: Chem. 2004, 223, 231-235. [CrossRef]

22. Xie, W.; Hu, P. Production of structured lipids containing medium-chain fatty acids by soybean oil acidolysis using SBA-15-pr- $\mathrm{NH}_{2}-\mathrm{HPW}$ catalyst in a heterogeneous manner. Org. Process Res. Dev. 2016, 20, 637-645. [CrossRef]

23. Gao, R.; Zhu, Q.; Dai, W.-L.; Fan, K. A green process for the epoxidation of dicyclopentadiene with aqueous $\mathrm{H}_{2} \mathrm{O}_{2}$ over highly efficient and stable HPW-NH $2-S B A-15$. RSC Adv. 2012, 2, 6087-6093. [CrossRef]

24. Karaki, M.; Karout, A.; Toufaily, J.; Rataboul, F.; Essayem, N.; Lebeau, B. Synthesis and characterization of acidic ordered mesoporous organosilica SBA-15: Application to the hydrolysis of cellobiose and insight into the stability of the acidic functions. J. Catal. 2013, 305, 204-216. [CrossRef]

25. Yuan, C.; Chen, J. Preparation of heterogeneous mesoporous silica-supported 12-tungstophosphoric acid catalyst and its catalytic performance for cyclopentene oxidation. Chin. J. Catal. 2011, 32, 1191-1198. [CrossRef]

26. Lei, J.; Chen, L.; Yang, P.; Du, X.; Yan, X. Oxidative desulfurization of diesel fuel by mesoporous phosphotungstic acid $/ \mathrm{SiO}_{2}$ : The effect of preparation methods on catalytic performance. J. Porous Mater. 2013, 20, 1379-1385. [CrossRef]

27. Singh, S.; Patel, A. Selective green esterification and oxidation of glycerol over 12-tungstophosphoric acid anchored to MCM-48. Ind. Eng. Chem. Res. 2014, 53, 14592-14600. [CrossRef]

28. Majda, D.; Napruszewska, B.D.; Zimowska, M.; Makowski, W. Porosity of SBA-15 after functionalization of the surface with aminosilanes. Microporous Mesoporous Mater. 2016, 234, 98-106. [CrossRef] 
29. Dong, X.; Wang, D.; Li, K.; Zhen, Y.; Hu, H.; Xue, G. Vanadium-substituted heteropolyacids immobilized on amine- functionalized mesoporous MCM-41: A recyclable catalyst for selective oxidation of alcohols with $\mathrm{H}_{2} \mathrm{O}_{2}$. Mater. Res. Bull. 2014, 57, 210-220. [CrossRef]

30. Chen, Y.; Cao, Y.; Suo, Y.; Zheng, G.-P.; Guan, X.-X.; Zheng, X.-C. Mesoporous solid acid catalysts of 12-tungstosilicic acid anchored to SBA-15: Characterization and catalytic properties for esterification of oleic acid with methanol. J. Taiwan Inst. Chem. Eng. 2015, 51, 186-192. [CrossRef]

31. Brahmkhatri, V.; Patel, A. 12-tungstophosphoric acid anchored to sba-15: An efficient, environmentally benign reusable catalysts for biodiesel production by esterification of free fatty acids. Appl. Catal. A 2011, 403, 161-172. [CrossRef]

32. Heykants, E.; Verrelst, W.H.; Parton, R.F.; Jacobs, P.A. Shape-selective zeolite catalysed synthesis of monoglycerides by esterification of fatty acids with glycerol. In Studies in Surface Science and Catalysis; Chon, H., Ihm, S.-K., Uh, Y.S., Eds.; Elsevier: New York, NY, USA, 1997; Volume 105, pp. 1277-1284.

33. Konwar, L.J.; Mäki-Arvela, P.; Kumar, N.; Mikkola, J.-P.; Sarma, A.K.; Deka, D. Selective esterification of fatty acids with glycerol to monoglycerides over $-\mathrm{SO}_{3} \mathrm{H}$ functionalized carbon catalysts. React. Kinet. Mech. Catal. 2016, 119, 121-138. [CrossRef]

34. Bossaert, W.D.; De Vos, D.E.; Rhijn, W.M.V.; Bullen, J.; Grobet, P.J.; Jacobs, P.A. Mesoporous sulfonic acids as selective heterogeneous catalysts for the synthesis of monoglyceides. J. Catal. 1999, 182, 156-164. [CrossRef]

35. Hermida, L.; Abdullah, A.Z.; Mohamed, A.R. Synthesis of monoglyceride through glycerol esterification with lauric acid over propyl sulfonic acid post-synthesis functionalized SBA-15 mesoporous catalyst. Chem. Eng. J. 2011, 174, 668-676. [CrossRef]

36. Pérez-Pariente, J.N.; Díaz, I.; Mohino, F.; Sastre, E. Selective synthesis of fatty monoglycerides by using functionalised mesoporous catalysts. Appl. Catal. A 2003, 254, 173-188. [CrossRef]

37. Liu, J.; Liu, Y.; Yang, W.; Guo, H.; Fang, F.; Tang, Z. Immobilization of phosphortungstic acid on amino-functionalized bimetallic Zr-La-SBA-15 and its highly catalytic performance for acetylation. J. Mol. Catal. A: Chem. 2014, 393, 1-7. [CrossRef]

38. Zhao, D.; Feng, J.; Huo, Q.; Melosh, N.; Fredrickson, G.H.; Chmelka, B.F.; Stucky, G.D. Triblock copolymer syntheses of mesoporous silica with periodic 50 to 300 angstrom pores. Science 1998, 279, 548-552. [CrossRef] [PubMed]

39. Wang, S.; Wang, K.; Dai, C.; Shi, H.; Li, J. Adsorption of $\mathrm{Pb}^{2+}$ on amino-functionalized core-shell magnetic mesoporous sba-15 silica composite. Chem. Eng. J. 2015, 262, 897-903. [CrossRef] 